\title{
Genetics of female functional virginity in the Parthenogenesis-Wolbachia infected parasitoid wasp Telenomus nawai (Hymenoptera: Scelionidae)
}

\author{
G Jeong ${ }^{1,3}$ and R Stouthamer ${ }^{2}$ \\ ${ }^{1}$ Laboratory of Entomology, Wageningen University, $6700 \mathrm{EH}$, Wageningen, The Netherlands; ${ }^{2}$ Department of Entomology, University of \\ California, Riverside, CA 92521, USA
}

\begin{abstract}
A lepidopteran egg parasitoid species Telenomus nawai consists of two distinct populations with different reproductive modes. One is a completely thelytokous population consisting of females only, whereas the other displays arrhenotokous reproduction where fertilized eggs develop into diploid females and unfertilized eggs into haploid males. Thelytoky in $T$. nawai is caused by a bacterial symbiont, the parthenogenesis-inducing (PI) Wolbachia. Recent theoretical studies have shown that when a $\mathrm{PI}$-Wolbachia is spreading in a population, mutations that allow uninfected females to produce more male offspring
\end{abstract}

will spread rapidly eventually becoming fixed. The consequence of such a mutation is that sexual reproduction is no longer successful in infected females. Here we determine the genetic basis of the females' inability to reproduce sexually by introgressing the genome of a thelytokous line into an arrhenotokous line. The results suggest that the mutations are recessive and inherited either as a single-locus major gene with some modifiers, or as two partially linked loci.

Heredity (2005) 94, 402-407. doi:10.1038/sj.hdy.6800617

Published online 3 November 2004

Keywords: female virginity mutations; mating reluctance; fertilization; parthenogenesis; Wolbachia; Telenomus nawai

\section{Introduction}

Heritable symbionts are prevalent among insects and cause a number of alterations in their host's reproduction (for reviews, see O'Neill et al, 1997; Bourtzis and Miller, 2003). These alterations range from a crossing incompatibility (cytoplasmic incompatibility), killing the male offspring (male killing), rendering genetic males into functional females (feminization) and inducing complete parthenogenetic reproduction or thelytoky (parthenogenesis induction).

Parthenogenesis induction appears to be limited to host taxa that normally have haplo-diploid sex determination or arrhenotoky, where unfertilized (haploid) eggs develop as males and fertilized (diploid) eggs as females. These arrhenotokous taxa include the bees and wasps (Hymenoptera), as well as a number of other taxa (Wrensch and Ebbert, 1993). Bacteria inducing parthenogenesis in their hosts have now been found in two bacterial taxa: Wolbachia (Huigens and Stouthamer, 2003) and Cardinium (Zchori-Fein et al, 2004). Parthenogenesisinducing (PI) Wolbachia manipulate the chromosome behavior in unfertilized infected eggs, by causing the first mitotic division to abort, resulting in the formation

Correspondence: $R$ Stouthamer, Department of Entomology, University of California, Riverside, CA 92521, USA.

E-mail: richard.stouthamer@ucr.edu

${ }^{3}$ Current address: Department of Entomology, University of California, Riverside, CA 92521, USA

Received 26 April 2004; accepted 30 September 2004; published online 3 November 2004 of a diploid nucleus and female development (Stouthamer and Kazmer, 1994). Infection with PI-Wolbachia in itself does not interfere with sexual reproduction: if infected eggs are fertilized, the PI-Wolbachia appears not to influence the chromosome behavior in the infected egg and, in these fertilized eggs, the paternal chromosome set participates in the formation of the infected female offspring (Stouthamer and Kazmer, 1994). The speed with which a PI infection in an arrhenotokous population will go to fixation is a function of (1) the sex ratio produced by the uninfected females, (2) the relative cost of being infected, (3) the transmission efficiency of the infection (Stouthamer, 1997) and (4) the presence of other sex ratio distorters that counteract the PI-infection such as a paternal sex ratio chromosome (Stouthamer et al, 2001).

A fifth factor that influences the infection frequency in populations was presented by Huigens and Stouthamer (2003). They argue that the highly female biased sex ratio, which results from a spreading PI-bacterium infection, selects for mutations that cause uninfected females to produce more male offspring. Owing to haplo-diploid sex determination, the way to achieve more male offspring is by increasing the number of unfertilized eggs that females produce. A complete male bias can be achieved when uninfected inseminated females do not fertilize their eggs or when uninfected females remain virgins. We refer to mutations that reduce the egg fertilization, as 'functional virginity' mutations. These mutations will spread from the uninfected population into the infected population, and from 
the infected back into the uninfected population when infected females produce some uninfected offspring through inefficient transmission of the Wolbachia infection. The end result of the presence of these mutations is that the infection goes to fixation, and that all the females in the population are homozygous for the 'functional virginity' mutations (Huigens and Stouthamer, 2003), and therefore they are no longer capable of producing offspring from fertilized eggs.

For most PI-Wolbachia-infected populations, it is possible to produce males by feeding the wasps antibiotics or by exposing the females to elevated rearing temperatures (Stouthamer, 1997). In most of these cases, the males and females of these populations are not capable of producing offspring sexually (Zchori-Fein et al, 1992; Arakaki et al, 2001; De Barro and Hart, 2001; Gottlieb and Zchori-Fein, 2001; Pannebakker, 2004). This lack of sexual reproduction may be caused by mutations that have accumulated in genes involved with sexual reproduction since the time that the population stopped reproducing sexually. Under this scenario, we would expect that both the male and the female sexual behavior would have been affected by mutations, and neither male nor female would be able to reproduce sexually any longer. However, consistent with the 'female functional virginity' hypothesis, in several cases it has been shown that only the females are no longer capable of sexual reproduction, while males are still able to successfully father offspring when they mate with females from a sexual population. For instance, in the wasp Apoanagyrus diversicornis, Pijls et al (1996) showed that males derived from the infected population were capable of fathering offspring with females from a closely related sexual form, while neither the males derived from the infected line nor those derived from the sexual form were capable of fathering offspring with the infected females. A similar pattern was found in the wasp Telenomus nawai (Arakaki et al, 2000). T. nawai has two distinctive modes of reproduction. One population originating from the island Okinawa is thelytokous, due to a Wolbachia infection (Arakaki et al, 2000), while the population collected on the large island reproduces by arrhenotoky. Arakaki et al (2000) reported that a cured sexual population could not be established from the (Wolbachia-infected) thelytokous population of $T$. nawai by antibiotic treatment. They found no evidence that the infected females used sperm when they mated; however, males from the infected population were capable of fathering offspring with females from the arrhenotokous population.

Here we study whether a mutation that prevents the establishment of a sexual population from the infected females exists in the Wolbachia-infected T. nawai population, and how this mutation is inherited. Our results show that the failure of sexual reproduction by females from the infected line is caused by recessive mutations and that only a few loci have a major effect.

\section{Materials and methods}

\section{Strains}

T. nawai (Hymenoptera: Scelionidae) is a parasitoid of moth eggs, in particular army worm species (Spodoptera) found in the Far East (Arakaki et al, 2000). The Wolbachia- infected thelytokous population originated from Ginowan, the island of Okinawa, Japan; the uninfected arrhenotokous population originated from Tsukuba, Ibaraki, on the large island of Japan. In our experiments, we used one infected isofemale line and one uninfected isofemale line. Both lines were reared on Mamestra brassicae (Lepidoptera: Noctuidae) eggs at $23 \pm 1^{\circ} \mathrm{C}$, $\mathrm{L}: \mathrm{D}=16: 8, \mathrm{R} . \mathrm{H} 60 \pm 10 \%$. In the experiments, we used egg cards containing 25-30 M. brassicae eggs to allow the wasps to produce offspring. To verify that the thelytokous T. nawai females were infected with Wolbachia, PCR with Wolbachia-specific primers (wsp) was performed as described in Braig et al (1998).

\section{Determination of genetic basis of the female functional virginity mutations}

The genetic basis of the female virginity trait can be detected by introgression of the thelytokous genome into the arrhenotokous line (Figure 1). To perform introgression, males were obtained from the thelytokous females by feeding them the antibiotic rifampicin in honey $(0.1 \%$ $\mathrm{v} / \mathrm{w}$ ) and rearing them at $30^{\circ} \mathrm{C}$. In all our crosses, we used wasps that were less than $24 \mathrm{~h}$ old. In preliminary experiments, we found that the mating success was higher in group matings than when single pairs were isolated in vials (data not shown). In general, the introgression experiments were performed as follows: females and males were isolated as pupae. These pupae were placed together in a vial and allowed to group-mate upon emergence. The males were left with the females for one day. Subsequently, the mated females were individually isolated, and for 5 days were daily given an egg card for offspring production. The offspring from each mother was sexed and counted. In the first generation of introgression, the mating group consisted of six arrhenotokous females (A) with 10 males derived from the thelytokous line $(\mathrm{T})$. In the next generation, we made a mating group consisting of nine $\mathrm{F}_{1}$ hybrid female offspring (AT) and 13 males (T) from the thelytokous line to generate $\mathrm{F}_{2}$ backcross females (ATT) (Figure 1). ATT backcross females inherit on average $\frac{1}{4}$ of their genome from the arrhenotokous line and $\frac{3}{4}$ from the thelytokous line. These $\mathrm{F}_{2}$ ATT females emerge together with males whose genome consists on average of $\frac{1}{2}$ the genome of the thelytokous line and $\frac{1}{2}$ the genome of the arrhenotokous

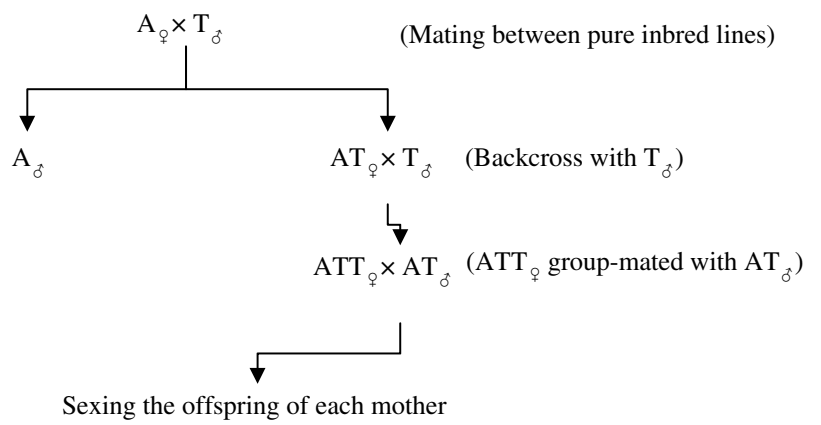

Figure 1 Mating scheme to determine the number of genes involved in the female virginity trait in the thelytokous line $\mathrm{A}=$ arrhenotokous genome, $\mathrm{T}=$ thelytokous genome, $\mathrm{AT}=$ hybrid offspring of arrhenotokous mother and thelytokous father, and ATT $=$ backcross offspring of AT hybrid mother and father derived from the thelytokous line. Note that $\mathrm{A}$ and $\mathrm{T}$ stand for genetic composition, not ploidy level. 
line. These males are indicated as AT males (Figure 1). We needed a large sample of ATT females to determine the offspring sex ratios that they produced; consequently, we allowed the ATT females to mate with their AT brothers, and after group mating for 1 day we treated them as above. To make sure that the AT males used in this experiment were equally capable of inseminating females as $\mathrm{T}$ males used in the first part of the introgression, we also group mated A females with AT males and determined the offspring sex ratio of these mated females (27 replicates). Several control crosses were done: 92 replicates of arrhenotokous female and male mating $\left(\mathrm{A}_{\varphi} \times \mathrm{A}_{\delta}\right)$. 45 replicates of the cross between arrhenotokous females and thelytokous males $\left(\mathrm{A}_{\varphi} \times \mathrm{T}_{\delta}\right)$ were made in addition to the ones described above, 54 replicates of the cross between hybrid females and arrhenotokous males $\left(\mathrm{AT}_{\varphi} \times \mathrm{A}_{\delta}\right)$ were made to determine if AT hybrid females mated equally well with A males as with T males.

\section{Do thelytokous females mate and use sperm to fertilize their eggs?}

A mating experiment was carried out to determine if the infected females mated at a lower frequency than arrhenotokous females. In all, 19 parasitized eggs from which arrhenotokous females would emerge and 19 eggs from which thelytokous females would emerge were put in a glass vial with 16 arrhenotokous males. After emergence, they were left in the vial to mate. After 1 day, the males were removed and the females were separated and were each given an egg card to parasitize. After 4 days, the females were examined for the presence of sperm in their spermathecae (magnification $\times 100$ ). Sperm can be seen in the spermatheca when it is removed from the abdomen. The remainder of the wasp was used to determine its Wolbachia infection status.

To test if inseminated thelytokous females produce hybrid offspring, we set up a thelytokous female with 10 arrhenotokous males and observed the mating. This female was allowed to produce offspring for 1 day and subsequently we determined if she carried sperm in her spermatheca. Her 14 offspring were used to determine if she had used the sperm to fertilize some of her eggs. The genetic make-up of the offspring was determined using an ISSR marker. The primer (sequences: 5'-GGA GAG GAG AGG AGA) yielded banding patterns that distinguished the thelytokous line from the arrhenotokous line. PCR reactions were performed using a temperature profile of $94^{\circ} \mathrm{C}$ for $2 \mathrm{~min}, 94^{\circ} \mathrm{C}$ for $1 \mathrm{~min}, 44.3^{\circ} \mathrm{C}$ for $1 \mathrm{~min}$ and $72^{\circ} \mathrm{C}$ for $2 \mathrm{~min}$ ( 35 cycles).

\section{Statistics}

To analyze the difference in offspring sex ratios between group-mated and individually mated females, a $\chi^{2}$ test was applied. For the analyses of offspring sex ratios, ANOVAs and Tukey's $b$ post hoc comparison tests were carried out after arcsin transformation of sex ratios for all crosses described in Table 1. All the analyses were performed with SPSS ver. 10.0.

\section{Results}

\section{Wolbachia infection status}

As previously determined by Arakaki et al (2000), the thelytokous line is infected with Wolbachia, while the arrhenotokous line is not.

\section{Determination of genetic basis of the female functional virginity trait}

The mean sex ratio of the cross $\mathrm{AT}_{\phi} \times \mathrm{A}_{\delta}$ was different from those produced by A females when mated with either A, T or AT males (Table1 and Figure 2a, b) (ANOVA $P<0.001$, Tukey's $b$ test), indicating that, in these hybrids there was some interaction between the $\mathrm{T}$ and $\mathrm{A}$ genomes or the presence of genes from the thelytokous line caused negative effect on female reproductive behaviors such as willingness to mate or utilizing sperm. The results of the $\mathrm{A}_{\phi} \times \mathrm{AT}_{\hat{\sigma}}$ cross showed that the AT males were functional, resulting in successful mating and female offspring production (Table 1). The AT males do not differ from either A or $\mathrm{T}$ males in their ability to fertilize females (ANOVA $P<0.001$, Tukey's $b$ test). We could therefore use the AT males in the cross with their ATT sisters. In the $\mathrm{ATT}_{\phi} \times \mathrm{AT}_{\hat{\delta}}$ cross, 86 out of 304 ATT females (28.3\%) who mated with their brothers do not produce female offspring (Table 2 and Figure 2c). A two-locus four-allele model would predict $25 \%$ of the females to fall in this class (see Table 2). Consequently, this model would fit reasonably well using only this class of females. However, we know more about the genetic make-up of the females under this model because $\frac{1}{4}$ of the mothers should fall in the class $\mathrm{AaBb}$, which is the same as the AT females. The distribution of offspring sex ratios for this class is known (see Figure 2b). In Figure 3 we partition out the distribution of the sex ratios produced by $\mathrm{AaBb}$ and aabb females under the assumption that each genotype will contain $\frac{1}{4}$ of all the females. Figure $3 a$ shows the total distribution of the offspring sex ratios. In Figure $3 b$ we show the expected distribution for the genotypes $\mathrm{AaBb}$ (which is the same genotype as AT

Table 1 Mean and standard deviation of the offspring produced in crosses between the experimental strains

\begin{tabular}{|c|c|c|c|c|c|c|}
\hline$q \times \hat{\sigma}$ & $\mathrm{N}$ & M. +9 & M. ठิ & M. O & $\%$ o & Total \\
\hline $\mathrm{A}_{\varphi} \times \mathrm{A}_{\hat{\sigma}}$ & 92 & $13.2 \pm 9.5$ & $3.7 \pm 4.8$ & $16.9 \pm 10.7$ & $22.3^{\mathrm{a}}$ & 1555 \\
\hline $\mathrm{A}_{\varphi} \times \mathrm{T}_{\hat{\delta}}$ & 45 & $20.2 \pm 10.4$ & $6.6 \pm 4.8$ & $26.9 \pm 12.0$ & $24.7^{\mathrm{a}}$ & 1210 \\
\hline $\mathrm{A}_{\varphi} \times \mathrm{AT}_{\hat{\delta}}$ & 27 & $16.7 \pm 11.7$ & $5.3 \pm 4.2$ & $22.0 \pm 13.6$ & $24.0^{\mathrm{a}}$ & 593 \\
\hline $\mathrm{AT}_{\varphi} \times \mathrm{A}_{\delta}$ & 54 & $16.4 \pm 9.1$ & $8.0 \pm 6.7$ & $24.4 \pm 12.2$ & $32.8^{\mathrm{b}}$ & 1319 \\
\hline $\mathrm{ATT}_{\phi} \times \mathrm{AT}_{\delta}$ & 304 & $10.9 \pm 13.1$ & $19.2 \pm 13.1$ & $30.4 \pm 12.6$ & $63.7^{c}$ & 9251 \\
\hline
\end{tabular}

N: number of replicates; M. $\$$ : mean number of female offspring; M. $\delta$ : mean number of male offspring; M. O: mean number of offspring; $\%$ $\delta$ : sex ratio; Total: total number of offspring. $\mathrm{A}=$ arrhenotokous line, $\mathrm{T}=$ thelytokous line, $\mathrm{AT}_{\phi}=$ offspring of arrhenotokous mother and thelytokous father, $\mathrm{AT}_{\delta}=$ male offspring of $\mathrm{AT}_{q}$, and $\mathrm{ATT}_{\phi}=$ offspring of $\mathrm{AT}$ mother and thelytokous father. $\% \hat{\mathrm{o}}$ with the same letter does not differ significantly (ANOVA $P<0.001$, Tukey's $b$ test). 
females) and for the genotype aabb (which is the same genotype as $\mathrm{T}$ females). When we subtract these two sets of females from the total distribution, then the remaining distributions should be for the genotypes $\mathrm{Aabb}$ and $\mathrm{aaBb}$
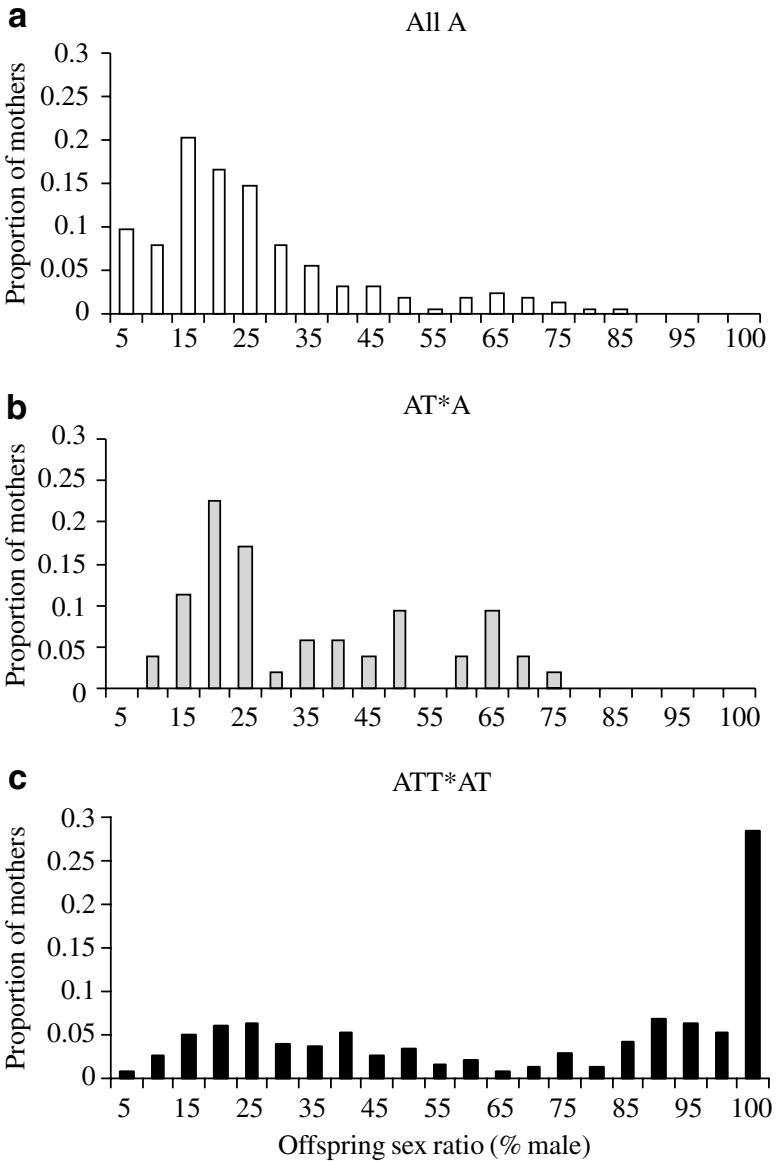

Figure 2 The offspring sex ratio produced by females with different levels of introgression of the thelytokous genome (T) into the arrhenotokous background (A). (a) Sum of all the crosses involving A females $\left(\mathrm{A}_{\varphi} \times \mathrm{A}_{\delta}, \mathrm{A}_{\varphi} \times \mathrm{T}_{\delta}, \mathrm{A}_{\varphi} \times \mathrm{AT}_{\hat{\sigma}}\right) ;(\mathbf{b})$ cross between AT females and A males; (c) cross between ATT females and AT males.
(Table 2 and Figure 3c). Figure 3c shows a bimodal distribution that could be consistent with the two different genotypes, where homozyogosity for one of the alleles has a much larger effect than the homozygosity of the other allele. Alternatively, if we assume a single-locus model and consider only the sex ratio classes not frequently found in the normal crosses with A females (ie those classes with offspring sex ratio's of larger than $85 \%$ males) as an expression of the homozygous T genotype, then about half of the ATT females (155 out of 304) fall in this class (Table 2). This is consistent with a single-locus model, but we would also have to assume some other minor modifier of the trait that results in the less than perfect expression of the trait.

\section{Do thelytokous females mate and use sperm to fertilize their eggs?}

Two females were lost during handling (one before given an egg card and the other after parasitization) and the spermatheca of one female could not be located. Sperm was found in the spermatheca of 10 out of 35 females and 17 out of 35 females were infected with Wolbachia. One of 17 infected females and nine of 18 arrhenotokous females had sperm in their spermathecae. The results show that occasionally the thelytokous females were inseminated, although the fraction of inseminated females was much lower than among arrhenotokous females. All of the 14 offspring of the one inseminated thelytokous female had only the ISSR banding pattern of the thelytokous line (data not shown). Therefore, she did not produce any daughters from fertilized eggs.

\section{Discussion}

Many genes are involved in sexual reproduction and mutations in any of them may influence the mating success and the fertilization proportion of the eggs in various ways. For instance, Silva and Stouthamer (1997) reported that in Trichogramma cordubensis (Hymenoptera: Trichogrammatidae), females from a Wolbachia-fixed population were not attractive to conspecific males, possibly resulting from a low or a complete lack of

Table 2 Comparison of two models to explain the genetics of the female functional virginity trait in the $\mathrm{ATT}_{\varphi} \times \mathrm{AT}_{\delta} \mathrm{cross}$

$\mathrm{AA}_{\varphi} \times \mathrm{a}_{0}$

$\mathrm{Aa}_{\odot} \times \mathrm{a}_{\text {}}$

Genotype of mates

$\mathrm{F}_{2}$

Genotype of female

Proportion

$\mathrm{F}_{3}$

Expected sex ratio

male

Expected number of mothers ${ }^{\mathrm{a}}$

Approximate number of mothers ${ }^{b}$

Normal
Model

1 locus 2 alleles

1 locus 2 alleles

loci 4 alleles

$\mathrm{AABB}_{\varphi} \times \mathrm{ab}^{\circ}$

$\mathrm{AaBb}_{\odot} \times \mathrm{ab}_{\text {ठํ }}$

$\begin{array}{ccc}\mathrm{Aa} & \mathrm{AaB} & \mathrm{AaBb} \\ \frac{1}{2} & \frac{1}{2} & \frac{1}{4}\end{array}$

Male biased $>80 \%$

152

155

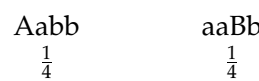

aabb

$\frac{1}{4}$

Normal or male biased

76

149
76

218
76
$100 \%$ male 76 86

In a single-locus two-alleles model, we assume that the functional virginity trait of the thelytokous line is recessive and is represented by a. In the two-loci four-alleles model, we assume that the recessive thelytokous genotype is aabb and that of the arrhenotokous line AABB.

${ }^{a}$ The variation in the sex ratios is continuous. The numbers of the mothers allocated in each genotype may not be precise and most likely largely overlap. Expected number of mothers is the number of mothers allocated to each genotype according to the model predictions.

${ }^{\mathrm{b}}$ Approximate number of mothers is the observed or estimated number of mothers in each genotype. 
406

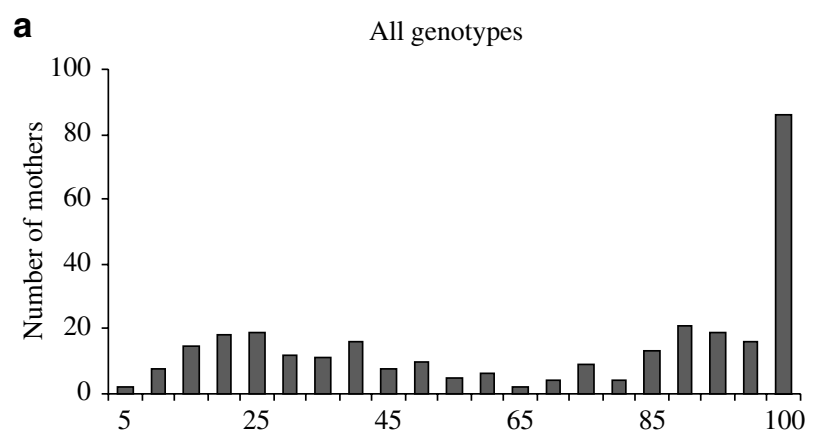

b

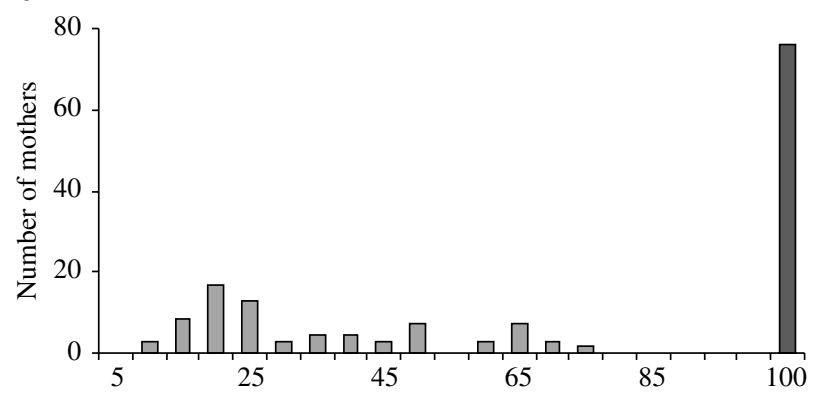

C

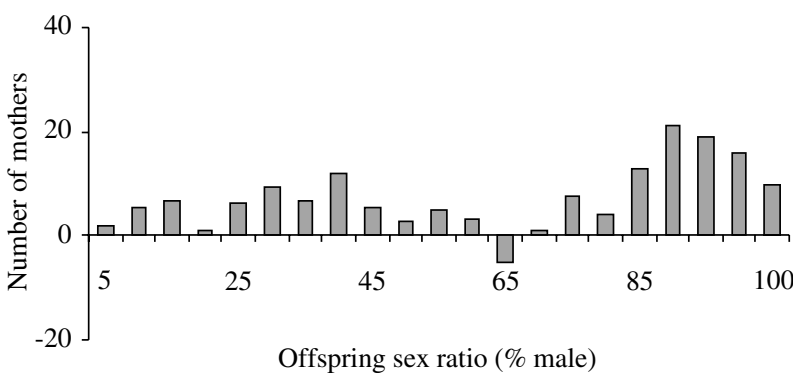

Figure 3 Sex ratio of offspring produced by ATT backcross females assuming as a two-loci four-allele model. Under this model, four different genotypes should be present in equal numbers of these mothers (Table 2). (a) The observed distribution of all mothers (genotypes); (b) the expected distribution of mothers with genotype $(\mathrm{AaBb})$ and genotype (aabb); (c) the expected distribution of mothers with genotypes (Aabb and $a a B b$ ) derived by subtracting distribution of (b) from (a).

pheromone production by the thelytokous females. In Encarsia formosa (Hymenoptera: Aphelinidae), females lost the function of spermatheca and males did not generate sperm (Zchori-Fein et al, 1992; Hunter, 1999). Similarly, in Leptopilina clavipes (Hymenoptera: Fitigidae) infected mated females cannot use sperm and antibiotics derived males are weakly functional (Pannebakker, 2004). In Muscidifurax uniraptor (Hymenoptera: Pteromalidae), at least three obstacles act on recovering sexual reproduction (Gottlieb and Zchori-Fein, 2001): females are reluctant to mate, a major muscle is absent from the spermstorage organ in females and males do not produce mature sperm.

The introgression experiments show that the female functional virginity trait of the thelytokous females follows a pattern consistent with simple Mendelian inheritance, controlled by a single locus with two alleles and some modifier influencing the complete expression, or by a two-locus four-allele model. At least two behaviors appear to be affected by the mutations: (1) thelytokous females mate at a much lower rate than the arrhenotokous ones, and (2) once inseminated they do not appear to fertilize any of the eggs.

It is often assumed that populations with haplodiploid sex determination carry a lower genetic load than diplo-diploid populations. This only applies to male-limited traits and to traits that are expressed in both males and females (Henter, 2003). In female-limited traits, the genetic load is similar to that in female-limited traits in diplo-diploid. Consequently, we expect that in normal sexual populations of these wasps, the females will carry some mutations in genes that influence their mating behavior or in genes involved in the chain that eventually results in eggs being fertilized. The relative frequency of mutations in such genes compared to other female-limited deleterious genes is most likely higher, simply because homozygosity for these traits will not affect their number of offspring but only the sex ratio of their offspring. In a sexual population that becomes infected with PI-bacteria, these female functional virginity mutations attain a selective advantage once the infection reaches high frequencies, eventually leading to the fixation of these traits. Those mutant forms that completely suppress sexual reproduction will eventually go to fixation. The fixation of the functional virginity mutations in the infected populations leads to an irreversible dependence of the reproduction of these lines on the presence of Wolbachia. Rare sexual reproduction in these lines is unlikely, apparently rare sex does occur occasionally in other species with thelytokous reproduction that is not associated with Wolbachia infection (Belshaw et al, 1999). The irreversibility of this trait would be expected to make the lineage vulnerable to the accumulation of mutations by Muller's ratchet (Muller, 1949).

As far as we know now, no sexual lines can be established from parthenogenetically reproducing species infected with Wolbachia outside Trichogramma species (Stouthamer et al, 1990). Elucidating the causal mechanisms of such failure can help understand the impacts of sex-manipulating symbionts on the host genome evolution. Why this type of functional virginity mutations has not spread in many Trichogramma populations remains to be determined. Possibly, their specific mating structure allows for the invasion with male-biased sex ratio distorters more readily than the mating structure of species where the infection has gone to fixation.

\section{Acknowledgements}

We thank Norio Arakaki and Hiroaki Noda for providing the experimental species. We are grateful to Ties Huigens and Fabrice Vavre for valuable suggestions on the experiments and to Leo Koopman, Andrea Gidding, and Frans van Aggelen for providing huge amount of M. brassicae eggs for the experiment. Isabel Silva, Bart Pannebakker, James Russell and Danel Vickerman are recognized for valuable comments on the manuscript. We thank two anonymous reviewers for valuable comments. This study is supported in part by NEXGEN Biotechnologies Inc. (Korea) to GJ and by an NSF FIBR award 0328363 to RS. 


\section{References}

Arakaki N, Miyoshi T, Noda H (2001). Wolbachia-mediated parthenogenesis in the predatory thrips Franklinothrips vespidormis (Thysanoptera: Insecta). Proc R Soc Lond B 268: 1011-1016.

Arakaki N, Noda H, Yamagishi K (2000). Wolbachia-induced parthenogenesis in the egg parasitoid Telenomus nawai. Entomol Exp Appl 96: 177-184.

Belshaw R, Quicke DLJ, Völkl W, Godfray HCJ (1999). Molecular markers indicate rare sex in a predominantly asexual parasitoid wasp. Evolution 53: 1189-1199.

Bourtzis K, Miller TA (eds) (2003). Insect Symbiosis. CRC Press: Boca Raton, FL.

Braig HR, Zhou W, Dobson SL, O'Neill SL (1998). Cloning and characterization of a gene encoding the major surface protein of the bacterial endosymbiont Wolbachia. J Bacteriol 180: 2373-2378.

De Barro PJ, Hart PJ (2001). Antibiotic curing of parthenogenesis in Eretmocerus mundus (Australian parthenogenic form). Entomol Exp Appl 99: 225-230.

Gottlieb Y, Zchori-Fein E (2001). Irreversible thelytokous reproduction in Muscidifurax uniraptor. Entomol Exp Appl 100: $271-278$.

Henter HJ (2003). Inbreeding depression and haplodiploidy: experimental measures in a parasitoid and comparisons across diploid and haplodiploid insect taxa. Evolution 57: 1793-1803.

Huigens ME, Stouthamer R (2003). Parthenogenesis associated with Wolbachia. In: Bourtzis K, Miller TA (eds) Insect Symbiosis. CRC Press: Boca Raton, FL, pp 247-266.

Hunter MS (1999). The influence of parthenogenesis-inducing Wolbachia on the oviposition behavior and sex-specific developmental requirements of autoparasitoid wasps. J Evol Biol 12: 735-741.

Muller H (1949). The Darwinian and modern conceptions of natural selection. Proc Am Philos Soc 93: 459-470.
O'Neill SL, Hoffmann AA, Werren JH (1997). Influential Passengers: Inherited Microorganisms and Arthropod Reproduction. Oxford University Press: New York.

Pannebakker B (2004). Evolutionary Consequences of Wolbachia Induced Parthenogenesis in the Parasitoid Leptopilina clavipes. PhD Thesis, Leiden University.

Pijls JWAM, Van Steenbergen HJ, Van Alphen JJM (1996). Asexuality cured: the relations and differences between sexual and asexual Apoanagyrus diversicornis. Heredity $\mathbf{7 6}$ 505-513.

Silva IMMS, Stouthamer R (1997). To mate or not to mate... Can sex pheromones be used as a taxonomic tool in Trichogramma spp.? Proc Exp Appl Entomol 8: 41-46.

Stouthamer R (1997). Wolbachia-induced parthenogenesis. In: O'Neill SL, Hoffmann AA, Werren JH (eds) Influential Passengers: Inherited Microorganisms and Arthropod Reproduction. Oxford University Press: New York, pp 102-124.

Stouthamer R, Kazmer DJ (1994). Cytogenetics of microbeassociated parthenogenesis and its consequence for gene flow in Trichogramma wasps. Heredity 73: 317-327.

Stouthamer R, Luck RF, Hamilton WD (1990). Antibiotics cause parthenogenetic Trichogramma (Hymenoptera/Trichogrammatidae) to revert to sex. Proc Natl Acad Sci USA 87: 2424-2427.

Stouthamer R, van Tilborg M, de Jong JH, Nunney L, Luck RF (2001). Selfish element maintains sex in natural populations of a parasitoid wasp. Proc R Soc Lond B 268: 617-622.

Wrensch DL, Ebbert MA (1993). Evolution and Diversity of Sex Ratio in Insects and Mites. Chapman \& Hall: New York and London.

Zchori-Fein E, Perlman SJ, Kelly SE, Katzir N, Hunter MS (2004). Characterization of a 'Bacteroidetes' symbiont in Encarsia wasps (Hymenoptera: Aphelinidae): proposal of 'Candidatus Cardinium hertigii'. Int J Syst Evol Microbiol 54 961-968.

Zchori-Fein E, Roush RT, Hunter MS (1992). Male production influenced by antibiotic treatment in Encarsia formosa, an asexual species. Experientia 48: 102-105. 